\title{
SWELLING OF A RUBBER BALL IN THE PRESENCE OF GOOD SOLVENT
}

\author{
By \\ Avner Friedman \\ and \\ Jianhua Zhang
}

IMA Preprint Series \# 974

May 1992 


\title{
SWELLING OF A RUBBER BALL IN THE PRESENCE OF GOOD SOLVENT*
}

\author{
AVNER FRIEDMAN* AND JIANHUA ZHANG†
}

\begin{abstract}
We consider the swelling of a 3-dimensional rubber ball in the presence of a good solvent. In Lagrangian coordinates the concentration $\varphi$ of rubber satisfies a diffusion equation with nonlinear nonlocal coefficients. In coordinates which are moving with the swollen rubber, $\varphi$ satisfies the porous medium equation $2 \varphi_{t}=\Delta \varphi^{2}$ with a moving boundary $r=s(t)$ on which $\varphi_{r}=-\dot{s}$ and $A \varphi_{r}+B \varphi=$ $B \theta \quad\left(A \geq 0, B \geq 0, A^{2}+B^{2}>0,0<\theta<1\right)$; for $A=0$, this problem also has a variational inequality formulation with a nonlinear parabolic operator. We prove existence, uniqueness and regularity of the solution and its free boundary, and determine its asymptotic behavior as $t \rightarrow \infty$.
\end{abstract}

$\S 1$. The problem. A rubber is a locally liquid polymer held together by crosslinks. When immersed in a good solvent such as gasoline it swells as much as several times its original size. The theory of equilibrium is based on minimizing the free energy function [4] [5] [13]. On the other hand the time dependent process of rubber swelling is not well understood [4]. One approach might possibly be based on the theory of mixture [2] [3], but this appears to be very complicated. A much simpler approach was recently developed by Rossi and Mazich [11] for the case of radially symmetric geometry, that is, for a swelling rubber ball: the rubber ball is immersed in a good solvent and is undergoing swelling.

It is assumed that the density of rubber $\rho_{R}$ and the density of solvent $\rho_{S}$ are constants (incompressibility assumption); for simplicity we take $\rho_{R}=\rho_{S}=1$. Then the concentrations and volume fractions coincide.

Set

$$
\begin{aligned}
& \phi_{R}=\text { volume fraction of rubber } \\
& \phi_{S}=\text { volume fraction of solvent. }
\end{aligned}
$$

Then

$$
\phi_{R}+\phi_{S}=1 .
$$

We fix the origin in the center of the ball, and denote the distance from the origin in the swelling coordinates by $r$, and in the original coordinates by $r_{0}$. We may view $r_{0}$ as a Lagrange variable and $r$ as an Euler variable. Initially (at $t=0$ ) the rubber is unswollen,

\footnotetext{
*This work is partially supported by the National Science Foundation Grant DMS-86-12880

*University of Minnesota, Institute for Mathematics and its Applications, Minneapolis, Minnesota 55455 .

$\nmid$ University of Minnesota, School of Mathematics, Minneapolis, Minnesota 55455.
} 
that is, $r=r_{0}$. As time evolves, the material point at $r_{0}$ will be displaced radially to position $r=r\left(r_{0}, t\right)$.

The ball initially occupies a region $r_{0} \leq a$.

We may consider $\phi_{R}$ as a function of either $\left(r_{0}, t\right)$ or $(r, t)$. The advantage of viewing it as a function of $\left(r_{0}, t\right)$ is that the spatial domain is then fixed, namely, it is the ball $r_{0} \leq a$.

\section{Initially}

$$
\phi_{R}\left(r_{0}, 0\right)=1 \quad \text { if } \quad r_{0}<a .
$$

A volume element $\Delta V\left(r_{0}, 0\right)=r_{0}^{2} d r_{0} d \sigma$ (where $d \sigma$ is a surface element on the unit sphere) initially located at $r=r_{0}$ will occupy volume $\Delta V\left(r_{0}, t\right)=r^{2} d r d \sigma \quad\left(r=r\left(r_{0}, t\right)\right)$ at time $t$, such that

$$
\phi_{R}\left(r_{0}, t\right) \Delta V\left(r_{0}, t\right)=\phi_{R}\left(r_{0}, 0\right) \Delta V\left(r_{0}, 0\right) .
$$

It follows that

$$
\phi_{R}\left(r_{0}, t\right) r^{2} d r=r_{0}^{2} d r_{0}
$$

or

$$
r^{3}\left(r_{0}, t\right)=\int_{0}^{r_{0}} \frac{3 y^{2} d y}{\phi_{R}(y, t)} .
$$

Conservation of mass of rubber and solvent gives ([11]; see also [7; Chap. 5])

$$
\frac{\partial \phi_{S}}{\partial t}=-\frac{\phi_{R}}{r^{2}} \frac{\partial}{\partial r}(\text { flux })
$$

We assume that the flux is given by Fick's law:

$$
\text { flux }=-\widetilde{D}\left(\phi_{S}\right) r^{2} \frac{\partial \phi_{S}}{\partial r}
$$

where $\widetilde{D}$ is a diffusion coefficient.

Substituting (1.6) into (1.5) and using (1.1), we find that $\phi_{R}$ satisfies the equation

$$
\frac{\partial \phi_{R}}{\partial t}=\frac{\phi_{R}}{r^{2}} \frac{\partial}{\partial r}\left(D\left(\phi_{R}\right) r^{2} \frac{\partial \phi_{R}}{\partial r}\right)
$$

where $D\left(\phi_{R}\right)=\widetilde{D}\left(1-\phi_{R}\right)$. 
For simplicity we shall make the assumption that $D\left(\phi_{R}\right)$ is a constant (see, however, Remark 7.2) and in fact take

$$
D\left(\phi_{R}\right)=1
$$

Using (1.3) in (1.7) and setting $\varphi=\phi_{R}\left(r_{0}, t\right)$, we then get

$$
\frac{\partial \varphi}{\partial t}=\frac{\varphi^{2}}{r_{0}^{2}} \frac{\partial}{\partial r_{0}}\left(\frac{r^{4}}{r_{0}^{2}} \varphi \frac{\partial \varphi}{\partial r_{0}}\right), \quad 0<r_{0}<a, t>0 .
$$

We make the assumption (which clearly must hold if $\varphi \in C^{1}$ ) that

$$
\frac{\partial \varphi(0, t)}{\partial r_{0}}=0, \quad t>0
$$

We recall the initial condition

$$
\varphi\left(r_{0}, 0\right)=1, \quad 0<r_{0}<a,
$$

and impose the boundary condition at $r_{0}=a$

$$
-A \frac{\partial \varphi}{\partial r}=B(\varphi-\theta) \quad \text { for } \quad r=r(a, t), t>0
$$

where $0<\theta<1$ and $A, B$ are nonnegative functions. The parameter $\theta$ arises from the Flory results [5] for the equilibrium concentration and it summarizes all polymer and network specific information of the rubbery material. In Sections $2-5$ we consider the special case $A=0, B=1$ :

$$
\varphi(a, t)=\theta, t>0
$$

where $0<\theta<1$. In Section 6 we extend the results to the general boundary condition at $r_{0}=a$. Finally, in Section 7 we consider the limiting case $\theta=0$.

\section{§2. Equivalent formulations.}

Definition 2.1. We refer to the system (1.9) - (1.12) as problem (P1).

Because of the relation (1.4), equation (1.9) is both nonlinear and nonlocal. Since, by the maximum principle, $\varphi \geq \theta$, the nonlinearity in $\varphi$ is nondegenerate.

In this section we transform (P1) into an equivalent problem by taking the independent variable to be $r=r\left(r_{0}, t\right)$ instead of $r_{0}$. For clarity we shall write

$$
\psi(r, t)=\varphi\left(r_{0}, t\right)
$$


We can rewrite (1.3) in the form

$$
\frac{\partial r}{\partial r_{0}}=\frac{r_{0}^{2}}{r^{2}} \frac{1}{\varphi\left(r_{0}, t\right)}=\frac{r_{0}^{2}}{r^{2}} \frac{1}{\psi(r, t)} .
$$

Differentiating (1.4) with respect to $t$ we get

$$
\begin{aligned}
\frac{\partial r}{\partial t} & =\frac{1}{r^{2}} \int_{0}^{r_{0}}\left(-\frac{y^{2}}{\varphi^{2}}\right) \varphi_{t} d y \\
& =\frac{1}{r^{2}} \int_{0}^{r_{0}}\left(-\frac{y^{2}}{\varphi^{2}}\right) \frac{\varphi^{2}}{y^{2}} \frac{\partial}{\partial y}\left(\frac{r^{4}}{y^{2}} \varphi \frac{\partial \varphi}{\partial y}\right) d y \quad \text { (by (1.9)) } \\
& =-\frac{r^{2}}{r_{0}^{2}} \varphi \frac{\partial \varphi}{\partial r_{0}} \quad(\text { by }(1.10)) \\
& =-\frac{r^{2}}{r_{0}^{2}} \psi \frac{\partial \psi}{\partial r} \frac{r_{0}^{2}}{r^{2}} \frac{1}{\psi} \quad(\text { by }(2.2)) .
\end{aligned}
$$

Hence

$$
\frac{\partial r}{\partial t}=-\frac{\partial \psi}{\partial r}
$$

Using (2.1), (2.2), we can transform (1.9) into

$$
\psi_{t}=\frac{1}{2}\left\{\left(\psi^{2}\right)_{r r}+\frac{2}{r}\left(\psi^{2}\right)_{r}\right\} .
$$

This equation is to be satisfied for $r<s(t), t>0$, where

$$
s(t)=r(a, t)
$$

$r=s(t)$ is the radius of the swelling rubber ball at time $t$. Note that

$$
\dot{s}=r_{t}(a, t)=-\psi_{r}(a, t), \quad \text { by }(2.3) .
$$

We can now reformulate problem (P1) as follows:

Problem (P2). Find functions $\psi(r, t)$ and $s(t)$, with $s(0)=a$, such that

$$
\begin{array}{lll}
\psi_{t}=\frac{1}{2} \Delta\left(\psi^{2}\right) & \text { if } & 0<r<s(t), t>0, \\
\frac{\partial \psi}{\partial r}(0, t)=0 & \text { if } & t>0, \\
\psi(r, 0)=1 & \text { if } & 0<r<a, \\
\psi(s(t), t)=\theta & \text { if } & t>0, \\
\dot{s}(t)=-\frac{\partial \psi}{\partial r}(s(t), t) & \text { if } & t>0,
\end{array}
$$


where $\Delta$ is the Laplacian in $\mathbf{R}^{3}$.

Problem (P2) is a free boundary problem. We expect $\psi_{r}$ to be negative (this, in fact, will be proved later on). Therefore the function

$$
v=\psi-\theta
$$

should be a positive function for $r<s(t)$, vanishing on the free boundary, whereas the free boundary $r=s(t)$ is monotone increasing. This observation suggests possibly another formulation of the problem in terms of a variational inequality, we shall now proceed to derive.

From (2.10) we get

$$
\frac{1}{2} \psi^{2}=\frac{1}{2} v^{2}+\theta v+\frac{1}{2} \theta^{2}=w+\frac{1}{2} \theta^{2}
$$

where

$$
w=\frac{1}{2} v^{2}+\theta v
$$

Introducing also the inverse function $m(r)=s^{-1}(t)$ of $r=s(t)$, we can rewrite (2.5)-(2.9) in the form

$$
\begin{array}{cll}
\frac{\partial}{\partial t} \sqrt{2 w+\theta^{2}}-\Delta w=0 & \text { if } & 0<r<\infty, t>m(r), \\
\frac{\partial w}{\partial r}(0, t)=0 & \text { if } & t>0, \\
w(r, 0)=\frac{1-\theta^{2}}{2} & \text { if } & 0<r<a, \\
w(r, m(r))=0 & \text { if } & a<r<\infty, \\
m_{r} w_{r}=-\theta & \text { on } & t=m(r), r>0, \\
m(r)=0 & \text { if } & 0<r<a .
\end{array}
$$

Observe that $w>0$ if $t>m(r)$, since $v>0$ if $t>m(r)$.

We now use the Duvaut transformation (cf. [6])

$$
W(r, t)= \begin{cases}\int_{m(r)}^{t} w(r, \tau) d \tau \quad \text { if } \quad t>m(r) \\ 0 \quad \text { if } \quad t<m(r), r>a .\end{cases}
$$


We easily compute that

$$
\begin{array}{lll}
\Delta W=\sqrt{2 W_{t}+\theta^{2}} \quad \text { if } & r>a, t>m(r), \\
\Delta W=\sqrt{2 W_{t}+\theta^{2}}-1 \quad \text { if } & 0<r<a, t>0
\end{array}
$$

and, trivially,

$$
\Delta W=\sqrt{2 W_{t}+\theta^{2}}-\theta \text { if } \quad r>a, t<m(r)
$$

(since $W=0$ in this set). Setting

$$
f(r)=\left\{\begin{array}{lll}
1 & \text { if } & 0<r<a \\
0 & \text { if } & r>a
\end{array}\right.
$$

we find that (2.12) reduces to the following variational inequality:

Problem (P3). Find $W(r, t)$ such that

$$
\begin{aligned}
& \sqrt{2 W_{t}+\theta^{2}}-\Delta W \geq f, \quad W \geq 0, \\
& \left(\sqrt{2 W_{t}+\theta^{2}}-\Delta W-f\right) W=0 \quad \text { a.e. if } \quad r>0, t>0
\end{aligned}
$$

and

$$
\begin{aligned}
& W_{r}(0, t)=0 \quad \text { if } \quad t>0, \\
& W(r, 0)=0 \quad \text { if } \quad r>0 .
\end{aligned}
$$

We can prove the existence of a solution using either one of the three formulations (P1), (P2), (P3). However, for proving regularity we prefer to use the formulations (P1) and (P2) (the nonlinearity of the parabolic operator in (2.14) causes technical difficulties), and for proving uniqueness formulation (P3) seems the most suitable.

$\S 3$. Existence and regularity. In this section we prove:

THEOREM 3.1. There exists a solution $\varphi$ of problem $(P 1)$ and a corresponding solution $(\psi, s)$ of problem (P2) such that

$$
\varphi \in C^{\infty}\left\{\left[0, r_{0}\right] \times(0, \infty)\right\}, \quad s \in C^{\infty}(0, \infty)
$$


furthermore, $\dot{s}(t)>0$ and $\partial \varphi / \partial r_{0}<0$ if $0<r_{0} \leq a, t>0$.

The function $\varphi$ is continuous at $\left(r_{0}, 0\right), 0 \leq r_{0}<a$ and at $(a, t), t>0$, but it is discontinuous at $(a, 0)$.

It will be convenient to work with the function $u=\varphi^{2}$ rather than with $\varphi$. Setting $x=r_{0}$ in problem (P1), we begin by approximating this problem by more regular $\varepsilon$ problems

$$
\begin{aligned}
& u_{t}=\frac{u^{3 / 2}}{x^{2}} \frac{\partial}{\partial x}\left(\frac{\sigma^{4}}{x^{2}} \frac{\partial u}{\partial x}\right), \varepsilon<x<a, t>0, \\
& u(a, t)=\theta^{2}, t>0 \\
& u_{x}(\varepsilon, t)=0, t>0 \\
& u(x, 0)=h_{\varepsilon}(x), \varepsilon<x<a
\end{aligned}
$$

where $\varepsilon$ is a small positive number,

$$
\sigma^{3}=\sigma^{3}(u)(x, t)=\int_{\varepsilon}^{x} \frac{3 y^{2} d y}{\sqrt{u(y, t)}}+\frac{\varepsilon^{3}}{\theta}
$$

and $h_{\varepsilon}(x)$ is a function in $C^{2+\alpha}[\varepsilon, a]$ such that

$$
h_{\varepsilon}(x)=1 \text { if } \varepsilon \leq x \leq a-2 \varepsilon, h_{\varepsilon}(x)=\theta^{2} \text { if } a-\varepsilon \leq x \leq a, \text { and } h_{\varepsilon}^{\prime}(x) \leq 0 .
$$

Set

$$
Q_{T}^{\varepsilon}=(\varepsilon, a) \times(0, T)
$$

We shall use the notation $u \in C^{2+\alpha, 1+\alpha / 2}$ to mean that the spatial derivatives $\nabla u, \nabla^{2} u$ and the time derivative $u_{t}$ are uniformly in $C^{\alpha}$ in the space variable and in $C^{\alpha / 2}$ in $t$. By $u \in C^{\beta, \beta / 2}$ we mean that $u$ is uniformly in $C^{\beta}$ in the space variable and in $C^{\beta / 2}$ in $t$; these and similar spaces are defined in [10].

Lemma 3.2. the system (3.1) has a solution $u_{\varepsilon}$ in $C^{2+\alpha, 1+\alpha / 2}\left(Q_{T}^{\varepsilon}\right)$ for some $\alpha \in(0,1)$.

Proof. For any $\lambda>0,0<\beta<1$, set

$$
B_{\lambda}=\left\{v \in C\left(\overline{Q_{T}^{\varepsilon}}\right), \quad \theta^{2} \leq v \leq 1, \quad\|v\|_{C^{\beta, \beta / 2}\left(Q_{T}^{\varepsilon}\right)} \leq \lambda\right\} .
$$


We define an operator $\mathcal{M}$ on $B_{\lambda}$ such that, for any $v \in B_{\lambda}, \quad u=\mathcal{M} v$ is the unique solution to

$$
\begin{aligned}
& u_{t}=\frac{v^{3 / 2}}{x^{2}} \frac{\partial}{\partial x}\left(\frac{\sigma^{4}(v)}{x^{2}} \frac{\partial u}{\partial x}\right), \varepsilon<x<a, 0<t<T \\
& u(a, t)=\theta^{2}, 0<t<T \\
& u_{x}(\varepsilon, t)=0,0<t<T \\
& u(x, 0)=h_{\varepsilon}(x), \varepsilon<x<a .
\end{aligned}
$$

By the maximum principle

$$
\theta^{2} \leq u \leq 1
$$

Since

$$
1 \leq \frac{\sigma^{3}(v)}{x^{3}} \leq \frac{1}{\theta},\left|\frac{\partial \sigma^{3}(v)}{\partial x}\right| \leq \frac{3 a^{2}}{\theta},
$$

we can apply the well known Hölder estimate for the uniformly parabolic equation in (3.1) (see [9]) to get

$$
\|u\|_{C^{\alpha, \alpha / 2}\left(Q_{T}^{\varepsilon}\right)} \leq C_{*} \quad \text { for some } \quad \alpha \in(0,1),
$$

where $C_{*}$ and $\alpha$ are constants depending on $\varepsilon$, but not on $\beta$ and $\lambda$. Choosing $\beta=\alpha$ and using (3.4), we can then apply the Schauder estimate to the equation in (3.1) to get

$$
\|u\|_{C^{2+\alpha, 1+\alpha / 2}\left(Q_{T}^{\varepsilon}\right)} \leq C
$$

where $C$ depends on $C_{*}$ and $\varepsilon$. Hence, if we choose $\lambda=C_{*}$ then $\mathcal{M}$ maps $B_{\lambda}$ into itself.

Using the estimate (3.5) one can easily deduce that $\mathcal{M}\left(B_{\lambda}\right)$ lies in a compact subset of $B_{\lambda}$ and $\mathcal{M}$ is a continuous mapping on $B_{\lambda}$, where $B_{\lambda}$ is endowed with the $C^{\alpha, \alpha / 2}$ topology. Hence, by the Schauder fixed point theorem, there exists a solution $u_{\varepsilon}$ of (3.1). Fix a small $\delta, 0<\delta \leq \frac{a}{4}$, and set

$$
\Omega_{T}^{\delta}=Q_{T}^{\delta} \backslash\{[a-\delta, a] \times[0, \delta]\} .
$$

By (3.3) and a priori Hölder estimates [9],

$$
\left\|u_{\varepsilon}\right\|_{C^{\alpha, \alpha / 2}\left(\Omega_{T}^{\delta}\right)} \leq C(\delta)
$$

for any $0<\varepsilon<\delta / 4$ where $\alpha \in(0,1)$ and $C(\delta)$ is a constant depending on $\delta$ but independent of $\varepsilon$. Using (3.6) we deduce, by the Schauder estimates, that

$$
\left\|u_{\varepsilon}\right\|_{C^{2+\alpha, 1+\alpha / 2}\left(\Omega_{T}^{\delta}\right)} \leq C(\delta)
$$

with another constant $C(\delta)$.

We next wish to estimate derivatives of $u_{\varepsilon}$ in $\varepsilon \leq x \leq \delta$. We begin by establishing bounds on the derivative $u_{\varepsilon, x}$. 
LEMмA 3.3. The solution $u_{\varepsilon}$ satisfies

$$
\frac{\partial}{\partial x} u_{\varepsilon}<0 \text { in } Q_{T}^{\varepsilon}
$$

Proof. Set $v=\partial u_{\varepsilon} / \partial x$. Differentiating the equation in (3.1) with respect to $x$ and dropping $\varepsilon$ in $u_{\varepsilon}$, we get

$$
\begin{aligned}
v_{t} & =\frac{\sigma^{4}}{x^{4}} u^{3 / 2} v_{x x}+\left[\frac{\partial}{\partial x}\left(\frac{\sigma^{4}}{x^{4}} u^{3 / 2}\right)+\frac{1}{x^{2}} u^{3 / 2} \frac{\partial}{\partial x}\left(\frac{\sigma^{4}}{x^{2}}\right)\right] v_{x} \\
& +\left[\frac{\partial}{\partial x}\left(\frac{u^{3 / 2}}{x^{2}} \frac{\partial}{\partial x}\left(\frac{\sigma^{4}}{x^{2}}\right)\right)\right] v
\end{aligned}
$$

where $\sigma=\sigma(u)$. Clearly also

$$
\begin{aligned}
& v(\varepsilon, t)=0, \quad 0<t<T, \\
& v(x, 0)=h_{\varepsilon}^{\prime}(x) \leq 0, \varepsilon<x<a,
\end{aligned}
$$

and

$$
v(a, t) \leq 0,0<t<T
$$

since $u$ takes its minimum at $x=a$. The assertion (3.8) now follows by applying the maximum principle to $v$.

LEMмA 3.4. The solution $u_{\varepsilon}$ satisfies

$$
\frac{\partial}{\partial x} u_{\varepsilon} \geq-A x \quad \text { in } \quad[\varepsilon, \delta] \times[0, T]
$$

where $A$ is a positive constant independent of $\varepsilon$ and $\delta$.

Proof. Setting $\varphi_{\varepsilon}=\sqrt{u_{\varepsilon}}$ and $\psi(r, t)=\varphi_{\varepsilon}\left(r_{0}, t\right)$ where $r_{0}=x$ and $r=r\left(r_{0}, t\right)$ is defined slightly differently than in (1.4), namely, $r=\sigma\left(u_{\varepsilon}\right)\left(r_{0}, t\right)$, one can easily find that $\psi$ still satisfies (2.5). Differentiating this equation with respect to $r$ we find that $\zeta=\psi_{r}$ satisfies

$$
\mathcal{L} \zeta \equiv \zeta_{t}-\psi \zeta_{r r}-\left(3 \psi_{r}+\frac{2}{r} \psi\right) \zeta_{r}-\left(\frac{2}{r} \psi_{r}-\frac{2}{r^{2}} \psi\right) \zeta=0
$$

Since $\partial r / \partial r_{0}>0$ (by $\left.(2.2)\right)$ and $\partial \varphi_{\varepsilon} / \partial r_{0} \leq 0$ (by Lemma 3.3), we have that $\psi_{r}<0$. Hence the coefficient of $\zeta$ in (3.11) is $\geq 0$, so that the maximum principle can be applied.

The function $\bar{\zeta} \equiv-A r$ is a subsolution to (3.11) since

$$
\mathcal{L} \bar{\zeta}=5 A \psi_{r}<0
$$


Using (3.7) with $\delta=a / 4$ we find that $\zeta=\psi_{r}$ majorizes $\bar{\zeta}$ on the parabolic boundary in the $(r, t)$-domain corresponding to $r_{0}=a / 4, r_{0}=\varepsilon$ and $t=0$, provided $A$ is sufficiently large. Hence $\psi_{r} \geq-A r$, from which (3.10) follows.

Remark 3.1. It seems difficult to prove (3.10) by working directly with (3.9); we can show that the coefficient of $v$ in (3.9) is $\leq 0$ (so that the maximum principle can be applied) only when $\theta \geq \frac{2}{3}$.

LEMma 3.5. There holds:

$$
\left\|u_{\varepsilon}\right\|_{C^{\alpha, \alpha / 2}\{[\varepsilon, 2 \delta] \times[0, T]\}} \leq \widetilde{C}
$$

where $\widetilde{C}$ and $\alpha$ are positive constants independent of $\varepsilon$ and $\delta$.

Proof. Rewrite the differential equation in (3.1), for $u=u_{\varepsilon}$, in the form

$$
u_{t}=\frac{\sigma^{4}}{x^{4}} u^{3 / 2} u_{x x}+f
$$

where

$$
f=\frac{4 \sigma}{x} u^{3 / 2}\left(\frac{1}{\sqrt{u}}-\frac{1}{2} \frac{\sigma^{3}}{x^{3}}\right) \frac{u_{x}}{x} .
$$

Recall that

$$
\theta^{2} \leq u \leq 1,1 \leq \frac{\sigma^{3}}{x^{3}} \leq \frac{1}{\theta}
$$

From (3.8), (3.10) we see that

$$
\left|\frac{u_{x}}{x}\right| \leq A
$$

Using also (3.16) we conclude that $f$ is bounded in $[\varepsilon, \delta] \times[0, T]$ uniformly with respect to $\varepsilon$ and $\delta$. Hence (3.14) is uniformly parabolic equation with uniformly bounded coefficients. By a priori Hölder estimates [9], the assertion (3.12) follows.

Proof of Theorem 3.1. Using (2.7) and (3.12), we can choose a sequence $u_{\varepsilon_{n}}$ which converges uniformly to a function $u$ in any region $\overline{\Omega_{T}^{\delta}}$, with $\delta \rightarrow 0$. It follows that $\psi=\sqrt{u}$ is a solution of $(1.9),(1.11),(1.12)$. From the estimate

$$
\left\|\frac{\partial}{\partial x} u_{\varepsilon_{n}}(x, t)\right\|_{L^{\infty}\left(\left[\varepsilon_{n}, \delta\right] \times[0, T]\right)} \leq A x \quad \text { for } \quad \delta=\frac{a}{4}
$$

derived above we conclude that

$$
\left|\frac{\partial u}{\partial x}(x, t)\right| \leq A x,
$$


so that (1.10) is also satisfied.

If we take a sequence of times $T=T_{n} \rightarrow \infty$ then, by the estimates established above for the corresponding solutions $u=u_{n}$ we can deduce that, for a subsequence, $u_{n}$ is convergent to a solution of (1.9) - (1.12) for all $t>0$.

By standard parabolic estimates, the corresponding solution $\psi$ is in $C^{\infty}$ for $0<r<$ $s(t), t>0$. Take $\delta \in(0, a)$ and denote by $\Psi(X, t) \quad\left(X \in \mathbf{R}^{3}\right)$ the solution of (2.5) in $\{|X|<\delta, t>0\}$ with $\Psi(X, t)=\psi(|X|, t)$ for $|X|=\delta$ and for $t=0$. Then, by standard regularity result for the nondegenerate parabolic equation $(2.5), \Psi(X, t)$ is $C^{\infty}$ in a neighborhood of $\{(O, t), t>0\}$ where $O$ is the origin in $\mathbf{R}^{3}$. By uniqueness, it is radially symmetric and therefore $\nabla_{X} \Psi(O, t)=0$. Then, again by uniqueness, $\Psi(X, t)=\psi(|X|, t)$. It follows that $\psi$ is in $C^{\infty}$ in a neighborhood of $\{(O, t) ; t>0\}$ where $O$ is the origin in $\mathbf{R}^{3}$. The $C^{2+\alpha, 1+\alpha / 2}$ regularity established for $u$ (by (3.7)) up to the free boundary, for $t \geq \delta$, implies that $\dot{s} \in C^{\alpha / 2}$ in $\{t \geq \delta\}$. From Lemma 3.3 we deduce that $\psi_{r}<0$ if $0<r<s(t)$ and, by the strong maximum principle for $\psi, \psi_{r}<0$ also on $r=s(t)$. Consequently $\dot{s}(t)>0$ for $t>0$.

To prove higher regularity for $s(t)$ and for $\psi$ at the free boundary, we can proceed similarly to the corresponding situation for the Stefan problem [12].

We have thus completed the proof of Theorem 3.1. Note that the solution $\varphi$ is not continuous at $(a, 0)$.

$\S 4$. Uniqueness. By a classical solution of (P1) we mean a solution $\varphi$ which is continuous in $\{[0, a] \times[0, \infty)\} \backslash(a, 0)$, with $\varphi_{r_{0}}$ continuous in $[0, a] \times(0, \infty)$. By the maximum principle it then follows that $\theta^{2} \leq \varphi \leq 1$ and $\partial \varphi / \partial r_{0}<0$ at $r_{0}=a$; therefore also $\dot{s}(t)>0$ if $t>0$.

Theorem 4.1. There exists at most one classical solution of (P1).

Proof. Suppose there exist two such solutions $\varphi$ and $\widehat{\varphi}$ with their corresponding $(\psi, s)$ and $(\widehat{\psi}, \widehat{s})$. Denote the corresponding solutions of (P3) by $W$ and $\widehat{W}$. It suffices to show that $W \equiv \widehat{W}$. If this is not true then we may assume that there exists a point $\left(r_{*}, t_{*}\right)$ with $t_{*}>0$ such that $W\left(r_{*}, t_{*}\right)>\widehat{W}\left(r_{*}, t_{*}\right)$. Denote by $G$ the largest connected open set in $[0, a] \times[0, \infty]$ which contains $\left(r_{*}, t_{*}\right)$ and on which $W>\widehat{W}$. Then

$$
\sqrt{2 W_{t}+\theta^{2}}-\Delta W=f, \sqrt{2 \widehat{W}_{t}+\theta^{2}}-\Delta \widehat{W} \geq f
$$

in $G$. It follows that

$$
\frac{2(W-\widehat{W})_{t}}{\sqrt{2 W_{t}+\theta^{2}}+\sqrt{2 \widehat{W}_{t}+\theta^{2}}}-\Delta(W-\widehat{W}) \leq 0 \text { in } G .
$$

Observe that $G$ must lie in $\{r<s(t)\}$ and thus $G_{T} \equiv G \cap\{t<T\}$ is a bounded set for any $T>0$. At each point on the parabolic boundary of $G$ either $W-\widehat{W}=0$ or (if $r=0$ 
at such a point) $(W-\widehat{W})_{r}=0$. Hence, by the maximum principle, $W-\widehat{W} \leq 0$ in $G_{T}$. If we take $T>t_{*}$ then we conclude that $W\left(r_{*}, t_{*}\right) \leq \widehat{W}\left(r_{*}, t_{*}\right)$, which is a contradiction.

Remark 4.1. One can prove the existence of a solution to problem (P3) by the penalty method. Introduce $\varepsilon$-family of $C^{\infty}$ functions $\beta_{\varepsilon}(\lambda)$ satisfying

$$
\begin{aligned}
& \beta_{\varepsilon}(\lambda)=0 \quad \text { if } \quad \lambda \geq \varepsilon, \quad \beta_{\lambda}(0)=-1, \\
& \beta_{\varepsilon}^{\prime}(\lambda) \geq 0, \beta_{\varepsilon}^{\prime \prime}(\lambda) \leq 0, \\
& \beta_{\varepsilon}(\lambda) \rightarrow-\infty \text { if } \lambda<0 \text { and } \varepsilon \downarrow 0 .
\end{aligned}
$$

Consider the parabolic problems:

$$
\begin{aligned}
& \sqrt{2 W_{t}+\theta^{2}}-\Delta W+\theta \beta_{\varepsilon}(W)=f_{\varepsilon}, 0<r<R, t>0, \\
& W_{r}(0, t)=0, \quad t>0, \\
& W(R, t)=0, \quad t>0, \\
& W(r, 0)=0, \quad 0<r<R
\end{aligned}
$$

where $f_{\varepsilon}$ is a smooth approximation to $f$ with $f_{\varepsilon}^{\prime} \leq 0$. Setting $v=2 W_{t}+\theta^{2}$ and differentiating (4.1) with respect to $t$, we get

$$
\frac{v_{t}}{\sqrt{v}}-\Delta v+\theta \beta_{\varepsilon}^{\prime}\left(\int_{0}^{t} \frac{1}{2}\left(v(r, \tau)-\theta^{2}\right) d \tau\right)\left(v-\theta^{2}\right)=0 .
$$

Clearly also

$$
\begin{aligned}
& v_{r}(0, t)=0, \quad t>0, \\
& v(R, t)=\theta^{2}, \quad t>0, \\
& v(r, 0)=\left(f_{\varepsilon}+\theta\right)^{2}, \quad 0<r<R .
\end{aligned}
$$

One can now prove, by a fixed point argument, that (4.5), (4.6) has a solution and this yields a solution $W_{\varepsilon}$ of $(4.1)$ - (4.4). (It seems harder to prove directly that the latter system has a solution.) Next one can apply the maximum principle to deduce that

$$
\theta^{2} \leq v \leq(1+\theta)^{2}
$$

Going back to $(4.1)-(4.4)$ we can proceed to derive a lower bound on $\beta_{\varepsilon}\left(W_{\varepsilon}\right)$ which is independent as $\varepsilon$ and $R$. It is then easy to show that for some subsequences $\varepsilon=\varepsilon_{n} \rightarrow$ $0, R=R_{n} \rightarrow \infty, W_{\varepsilon}$ converges to a solution $W$ of (P3) (cf. [8] where this is done for the Stefan problem). However, it seems difficult to establish regularity of the free boundary by working directly with the variational inequality formulation. 
$\S 5$. Asymptotic behavior as $\boldsymbol{t} \rightarrow \infty$. If the solution $W$ of (P3) converges to a limit $W_{0}$ as $t \rightarrow \infty$, then formally $W_{0}$ is the solution to the elliptic variational inequality

$$
\begin{gathered}
-\Delta W_{0} \geq f-\theta, W_{0} \geq 0, \\
\left(-\Delta W_{0}-f+\theta\right) W=0 \text { a.e. in }\{r>0\} \\
\frac{\partial}{\partial r} W_{0}(0)=0 .
\end{gathered}
$$

Since $f-\theta=-\theta$ if $r>a, W_{0}$ must have compact support (see [6; p. 9]). In fact, we can compute $W_{0}$ explicitly:

$$
W_{0}(r)=\left\{\begin{array}{l}
-\frac{1-\theta}{6} r^{2}+\frac{a^{2}}{2}\left(1-\theta^{1 / 3}\right), \quad 0<r<a \\
\frac{\theta}{6} r^{2}+\frac{a^{3}}{3 r}-\frac{a^{2} \theta^{1 / 3}}{2}, \quad a<r<\rho \\
0, \quad r>\rho
\end{array}\right.
$$

where

$$
\rho=\frac{a}{\theta^{1 / 3}} .
$$

In order to compare $W$ with $W_{0}$, let us introduce the principal eigenvalue $\lambda$ and a corresponding eigenfunction $\zeta(r)$ of

$$
\begin{gathered}
\Delta \zeta+\lambda \zeta=0 \quad \text { in } \quad 0<r<\rho, \\
\zeta_{r}(0)=0, \quad \zeta(\rho)=0, \\
\zeta(r)>0 \quad \text { if } \quad 0<r<\rho .
\end{gathered}
$$

ThEOREM 5.1. (i) For all $r>0, t>0$,

$$
W(r, t) \leq W_{0}(r) ;
$$

(ii) There exists a positive constant $K$ such that

$$
W_{0}(r)-W(r, t) \leq K \zeta(r) e^{-\lambda \theta t}
$$


for all $0<r<\rho, t>0$.

Proof. The function $W(r, t)$, for each fixed $t$, satisfies the same variational inequality as $W_{0}$ but with $f-\theta$ replaced by

$$
\widehat{f} \equiv f-\sqrt{2 W_{t}+\theta^{2}} .
$$

Since $W_{t} \geq 0, \widehat{f} \leq f-\theta$ and therefore, by a comparison theorem for variational inequalities (see [6; Chap. 1]), $W(r, t) \leq W_{0}(r)$.

To prove (5.7) set

$$
L v \equiv \frac{1}{\theta} v_{t}-\Delta v .
$$

The function $v=W_{0}-W$, in $\{0<r<\rho, t>0\}$, satisfies

$$
\begin{aligned}
L v & =-\frac{1}{\theta} W_{t}-\Delta W_{0}+\Delta W=-\frac{1}{\theta} W_{t}+f-\theta+\Delta W \\
& \leq-\frac{1}{\theta} W_{t}+f-\theta+\left(-f+\sqrt{2 W_{t}+\theta^{2}}\right) \quad \text { (by } \\
& =-\left(\frac{1}{\theta} W_{t}+\theta\right)+\sqrt{2 W_{t}+\theta^{2}} \leq 0
\end{aligned}
$$

since $W_{t} \geq 0$.

On the boundary:

$$
\begin{array}{ll}
\zeta_{r}=v_{r}=0 \quad, \quad r=0, t>0, \\
\zeta=v=0 \quad, \quad r=\rho, t>0
\end{array}
$$

and, if $K$ is large enough,

$$
v(r, 0)=W_{0}(r)-W(r, 0) \leq K \zeta(r), \quad 0<\rho<a .
$$

Since

$$
L\left[K \zeta(r) e^{-\lambda \theta t}\right]=0,
$$

we can apply the maximum principle to $v-K \zeta e^{-\lambda \theta t}$ to deduce the assertion (5.7).

COROllary 5.2. The free boundary satisfies:

$$
s(t) \uparrow \frac{a}{\theta^{1 / 3}} \quad \text { as } \quad t \uparrow \infty .
$$


$\S 6$. More general boundary conditions. In this section we replace the boundary condition (1.12) by the flux condition

$$
-A \frac{\partial \varphi}{\partial r}=B(\varphi-\theta) \quad \text { for } \quad r=r(a, t), t>0,
$$

where $\theta$ is a constant, $0<\theta<1$, and $A, B$ are smooth functions of $(r, t)$, with $A>0, B \geq 0$. For simplicity we shall take $A$ and $B$ to be constants; see however Remark 6.3.

In view of $(2.2)$, we can rewrite this flux condition in the form

$$
\frac{A r^{2} \varphi}{a^{2}} \frac{\partial \varphi}{\partial r_{0}}+B \varphi=B \theta \quad \text { for } \quad r_{0}=a, \quad t>0 .
$$

Definition. If in problem (P1) we replace (1.12) by (6.1), then we refer to the new problem as problem $\left(\mathrm{P} 1^{\prime}\right)$. Similarly we define problem $\left(\mathrm{P} 2^{\prime}\right)$ whereby $(2.8)$ is replaced by

$$
A \frac{\partial \psi}{\partial r}+B \psi=B \theta \quad \text { for } \quad r=s(t), \quad t>0 .
$$

THEOREM 6.1. There exists a solution to problem $\left(P 1^{\prime}\right)$ having the same regularity as the solution established for problem $(P 1)$ in Theorem 3.1; furthermore, $\partial \varphi / \partial r_{0}<0$ if $0<r_{0} \leq a, t>0$ and

$$
\varphi_{r_{0}} \in L^{\infty}((0, a) \times(0, \infty)), \varphi \in C^{\alpha}([0, a] \times[0, \infty))
$$

for some $\alpha \in(0,1)$.

Proof. As in $\S 3$ we first consider the $\varepsilon$-approximate problem (with (1.12) replaced by (6.1)) for which we can prove existence of a solution $\varphi_{\varepsilon}$ as well as the estimate

$$
\theta \leq \varphi_{\varepsilon}\left(r_{0}, t\right) \leq 1 \text {. }
$$

We can choose the initial data for the $\varepsilon$-approximate problem such that $\zeta=\partial \varphi_{\varepsilon} / \partial r_{0}$ is continuous at $(a, 0)$ and, at the same time, it is nonpositive and bounded on $\{t=0\}$ independently of $\varepsilon$. Applying the maximum principle to $\zeta$ (cf. (3.11)) we conclude that

$$
-C \leq \zeta \leq 0
$$

where $C$ is a constant independent of $\varepsilon$.

From (2.9) and (6.4), (6.5) we deduce that $0 \leq \dot{s} \leq C$. Using this and (6.5) we can then derive (as in [10]; Chap. 10) Hölder estimate for $\psi_{\varepsilon}$, and hence also for $\varphi_{\varepsilon}$, in a neighborhood of $(a, 0)$, with Hölder exponent and coefficient independent of $\varepsilon$. finally, by following the arguments in the proof of Theorem 3.1 we can complete the proof of Theorem 6.1 as we let $\varepsilon \rightarrow 0$. 
Corollary 6.2. There exists a solution $(\psi, s)$ to problem $\left(P 2^{\prime}\right)$ with $\dot{s}(t)>0$ if $t>0$; furthermore,

$$
\begin{gathered}
s \in C^{1+\alpha}[0, \infty), \\
s \in C^{\infty}(0, \infty) .
\end{gathered}
$$

Indeed, (6.6) follows from (6.2), (2.9) and the Hölder continuity of $\psi$, whereas the proof of (6.7) is similar to the corresponding proof in the case of (1.12).

By a classical solution to problem $\left(\mathrm{P}^{\prime}\right)$ we mean a solution $\varphi$ which satisfies (6.3). The next theorem establishes uniqueness for such solutions.

THEOREM 6.3. There exists at most one classical solution to problem $\left(P 1^{\prime}\right)$.

Remark 6.1. Problem (P1') cannot be recast as a variational inequality; consequently the uniqueness proof for problem (P1) cannot be extended to the present case. Note however that the classical solution to problem $\left(\mathrm{P} 1^{\prime}\right)$ is more regular at $(a, 0)$ than the solution to problem (P1) (the solution to $(\mathrm{P} 1)$ is discontinuous at $(a, 0)$ ). This additional regularity will enable us to work directly with the formulation (P1) (something we are unable to do in the case of problem (P1)).

Proof. Suppose $\widehat{\varphi}$ and $\varphi$ are two solutions and set

$$
\widehat{u}(x, t)=\widehat{\varphi}^{2}\left(r_{0}, t\right), u(x, t)=\varphi^{2}\left(r_{0}, t\right), \quad x=r_{0} .
$$

Then both $\widehat{u}$ and $u$ satisfy

$$
\begin{gathered}
v_{t}=\frac{v^{3 / 2}}{x^{2}} \frac{\partial}{\partial x}\left(\frac{\sigma^{4}}{x^{2}} v_{x}\right), \quad 0<x<a, \quad t>0, \\
v_{x}(0, t)=0, \quad t>0 \\
\frac{A}{2 a^{2}} \sigma^{2} v_{x}+B v^{1 / 2}=B \theta, \quad x=a, \quad t>0, \\
v(x, 0)=1, \quad 0 \leq x<a
\end{gathered}
$$

with

$$
\begin{aligned}
& \sigma^{3}=\widehat{r}^{3}=\int_{0}^{x} \frac{3 y^{2}}{\sqrt{\widehat{u}(y, t)}} d y \text { for } \widehat{u}, \\
& \sigma^{3}=r^{3}=\int_{0}^{x} \frac{3 y^{2}}{\sqrt{\widehat{u}(y, t)}} d y \text { for } u .
\end{aligned}
$$

Set $w=\widehat{u}-u$. 
LEMmA 6.4. There holds:

$$
\left|\widehat{r}^{4}-r^{4}\right| \leq C x \int_{0}^{x}|w| 3 y^{2} d y .
$$

Proof. Using the estimates

$$
\begin{aligned}
& \theta^{2} \leq \widehat{u}, u \leq 1 \\
& x^{3} \leq \widehat{r}^{3}, r^{3} \leq \frac{x^{3}}{\theta}
\end{aligned}
$$

we have

$$
\begin{aligned}
& \left|\widehat{r}^{4}-r^{4}\right|=\left|\left(\widehat{r}^{3}\right)^{4 / 3}-\left(r^{3}\right)^{4 / 3}\right| \\
& =\left|\int_{0}^{1} \frac{4}{3}\left[r^{3}+\eta\left(\widehat{r}^{3}-r^{3}\right)\right]^{1 / 3} d \eta\right| \cdot\left|\widehat{r}^{3}-r^{3}\right| \\
& \leq C x\left|\int_{0}^{x}\left(\frac{1}{\sqrt{\hat{u}}}-\frac{1}{\sqrt{u}}\right) 3 y^{2} d y\right| \leq C x \int_{0}^{x}|\widehat{u}-u| 3 y^{2} d y .
\end{aligned}
$$

Lemma 6.5. For any $\varepsilon>0$ there is a positive constant $C(\varepsilon)$ such that

$$
\int_{0}^{T} \int_{0}^{a} \frac{1}{x^{4}}\left(\int_{0}^{x}|w| 3 y^{2} d y\right)^{2} d x d t \leq \varepsilon \int_{0}^{T} \int_{0}^{a} x^{2} w_{x}^{2}+C(\varepsilon) \int_{0}^{T} \int_{0}^{a} x^{2} w^{2} .
$$

for any $0<T<\infty$.

Proof. By Schwarz's inequality, the left-hand side of (6.14) is bounded by

$$
\begin{aligned}
& \int_{0}^{T} \int_{0}^{a} \frac{1}{x}\left(\int_{0}^{x} w^{2} 3 y^{2} d y\right) d x d t \\
& =\int_{0}^{T} \log x \cdot\left[\int_{0}^{x} w^{2} 3 y^{2} d y\right]_{x=0}^{x=a} d t \\
& \quad-\int_{0}^{T} \int_{0}^{a} \log x \cdot w^{2} 3 x^{2} d x d t \quad \text { (by integration by parts) }
\end{aligned}
$$




$$
\begin{aligned}
\leq 3|\log a| & \int_{0}^{T} \int_{0}^{a} x^{2} w^{2} d x d t+\int_{0}^{T}\left[\left(\frac{x^{3}}{3}-x^{3} \log x\right) w^{2}\right]_{x=0}^{x=a} d t \\
& +\int_{0}^{T} \int_{0}^{a} 2 w_{x} w\left(x^{3} \log x-\frac{x^{3}}{3}\right) d x d t \quad \text { (by integration by parts). }
\end{aligned}
$$

Since $\left|x^{3} \log x-x^{3} / 3\right| \leq C x^{2}$,

$$
\int_{0}^{T}\left|w_{x} w\right| x^{2} \leq \varepsilon \int_{0}^{T} \int_{0}^{a} x^{2} w_{x}^{2}+C(\varepsilon) \int_{0}^{T} \int_{0}^{a} w^{2},
$$

and

$$
\int_{0}^{T} w^{2}(a, t) d t \leq \varepsilon \int_{0}^{T} \int_{0}^{a} x^{2} w_{x}^{2}+C(\varepsilon) \int_{0}^{T} \int_{0}^{a} w^{2}
$$

the assertion (6.14) follows.

Subtracting the equation (6.8) for $u$ from the one for $\widehat{u}$, then multiplying by $w$ and integrating, we get

$$
\begin{aligned}
\int_{0}^{T} \int_{0}^{a} x^{2} w_{t} w= & \int_{0}^{T} \int_{0}^{a}\left[\widehat{u}^{3 / 2} \frac{\partial}{\partial x}\left(\frac{\widehat{r}^{4}}{x^{2}} \widehat{u}_{x}\right)-u^{3 / 2} \frac{\partial}{\partial x}\left(\frac{r^{4}}{x^{2}} u_{x}\right)\right] w \\
= & \int_{0}^{T} \int_{0}^{a} \frac{\partial}{\partial x}\left[\frac{\widehat{r}^{4}}{x^{2}} \widehat{u}^{3 / 2} \widehat{u}_{x}-\frac{r^{4}}{x^{2}} u^{3 / 2} u_{x}\right] w \\
& -\int_{0}^{T} \int_{0}^{a}\left[\frac{\widehat{r}^{4}}{x^{2}} \widehat{u}_{x} \frac{3}{2} \sqrt{\widehat{u}} \widehat{u}_{x}-\frac{r^{4}}{x^{2}} u_{x} \frac{3}{2} \sqrt{u} u_{x}\right] w \\
= & \int_{0}^{T}\left[\left(\frac{\widehat{r}^{4}}{x^{2}} \widehat{u}^{3 / 2} \widehat{u}_{x}-\frac{r^{4}}{x^{2}} u^{3 / 2} u_{x}\right) w\right]_{x=0}^{x=a}-\int_{0}^{T} \int_{0}^{a}\left(\frac{\widehat{r}^{4}}{x^{2}} \widehat{u}^{3 / 2} \widehat{u}_{x}-\frac{r^{4}}{x^{2}} u^{3 / 2} u_{x}\right) w_{x} \\
& -\frac{3}{2} \int_{0}^{T} \int_{0}^{a}\left(\frac{\widehat{r}^{4}}{x^{2}} \sqrt{\widehat{u}} \widehat{u}_{x}^{2}-\frac{r^{4}}{x^{2}} \sqrt{u} u_{x}^{2}\right) w \equiv I_{1}-I_{2}-\frac{3}{2} I_{3} .
\end{aligned}
$$

From (6.10) we deduce that 


$$
\left(\widehat{r}^{2} \widehat{u}_{x}-r^{2} u_{x}\right)(\widehat{u}-u) \leq 0 \quad \text { at } \quad x=a .
$$

We now proceed to estimate the terms $I_{j}$. First

$$
\begin{aligned}
I_{1} & =\frac{1}{a^{2}} \int_{0}^{T}\left[\left(\widehat{r}^{4} \widehat{u}^{3 / 2} \widehat{u}_{x}-r^{4} u^{3 / 2} u_{x}\right) w\right](a, t) d t \\
& =\frac{1}{a^{2}} \int_{0}^{T}\left[\widehat{r}^{2} \widehat{u}^{3 / 2}\left(\widehat{r}^{2} \widehat{u}_{x}-r^{2} u_{x}\right) w\right](a, t) d t+\frac{1}{a^{2}} \int_{0}^{T}\left[\widehat{r}^{2}\left(\widehat{u}^{3 / 2}-u^{3 / 2}\right) r^{2} u_{x} w\right](a, t) d t \\
& +\frac{1}{a^{2}} \int_{0}^{T}\left[\left(\widehat{r}^{2}-r^{2}\right) u^{3 / 2} r^{2} u_{x} w\right](a, t) .
\end{aligned}
$$

The first term on the right-hand side is $\leq 0$ by (6.17). The second term is bounded by $C \int_{0}^{T} w^{2}(a, t) d t$. The third term can be estimated (using Lemma 6.4) by

$$
C \int_{0}^{T} \int_{0}^{a}|w| 3 y^{2}|w(a, t)| d y d t
$$

which is bounded by

$$
C \int_{0}^{T} \int_{0}^{a} x^{2} w^{2}+C \int_{0}^{T} w^{2}(a, t) .
$$

Recalling (6.15) we conclude that

$$
I_{1} \leq \varepsilon \int_{0}^{T} \int_{0}^{a} x^{2} w_{x}^{2}+C(\varepsilon) \int_{0}^{T} \int_{0}^{a} x^{2} w^{2} .
$$

Next, we can write

$$
\begin{aligned}
-I_{2}= & -\int_{0}^{T} \int_{0}^{a} \frac{\widehat{r}^{4}}{x^{2}} \widehat{u}^{3 / 2} w_{x}^{2}-\int_{0}^{T} \int_{0}^{a}\left(\frac{\widehat{r}^{4}}{x^{2}} \widehat{u}^{3 / 2}-\frac{r^{4}}{x^{2}} u^{3 / 2}\right) u_{x} w_{x} \\
\leq & -\theta^{3} \int_{0}^{T} \int_{0}^{a} x^{2} w_{x}^{2}-\int_{0}^{T} \int_{0}^{a} \frac{\widehat{r}^{4}}{x^{2}}\left(\widehat{u}^{3 / 2}-u^{3 / 2}\right) u_{x} w_{x} \\
& -\int_{0}^{T} \int_{0}^{a}\left(\frac{\widehat{r}^{4}}{x^{2}}-\frac{r^{4}}{x^{2}}\right) u^{3 / 2} u_{x} w_{x}
\end{aligned}
$$


where (6.13) was used. The second integral on the right-hand side is estimated, by the mean value theorem, by

$$
C \int_{0}^{T} \int_{0}^{a} x^{2}\left|w w_{x}\right|
$$

and the third integral is estimated, using Lemma 6.4 , by

$$
\int_{0}^{T} \int_{0}^{a} \frac{1}{x^{2}} \cdot C x\left(\int_{0}^{x}|w| 3 y^{2} d y\right) \cdot\left|u_{x}\right|\left|w_{x}\right| .
$$

Hence

$$
\begin{aligned}
-I_{2} & \leq-\theta^{3} \int_{0}^{T} \int_{0}^{a} x^{2} w_{x}^{2}+\varepsilon^{\prime} \int_{0}^{T} \int_{0}^{a} x^{2} w_{x}^{2}+C\left(\varepsilon^{\prime}\right) \int_{0}^{T} \int_{0}^{a} x^{2} w^{2} \\
& +C\left(\varepsilon^{\prime}\right) \int_{0}^{T} \int_{0}^{a} \frac{1}{x^{4}}\left(\int_{0}^{x}|w| 3 y^{2} d y\right)^{2},
\end{aligned}
$$

for any small $\varepsilon^{\prime}>0$.

Applying Lemma 6.5 with $\varepsilon \ll 1 / C\left(\varepsilon^{\prime}\right)$, we conclude that

$$
-I_{2} \leq-\frac{1}{2} \theta^{3} \int_{0}^{T} \int_{0}^{a} x^{2} w_{x}^{2}+C \int_{0}^{T} \int_{0}^{a} x^{2} w^{2}
$$

where $C$ is a large constant (depending on $\theta$ ).

Finally,

$$
\begin{aligned}
-I_{3} & =\int_{0}^{T} \int_{0}^{a} \frac{\widehat{r}^{4}}{x^{2}} \sqrt{\widehat{u}}\left(\widehat{u}_{x}^{2}-u_{x}^{2}\right) w+\int_{0}^{T} \int_{0}^{a} \frac{\widehat{r}^{4}}{x^{2}}(\sqrt{\widehat{u}}-\sqrt{u}) u_{x}^{2} w \\
& +\int_{0}^{T} \int_{0}^{a}\left(\frac{\widehat{r}^{4}}{x^{2}}-\frac{r^{4}}{x^{2}}\right) \sqrt{u} u_{x}^{2} w .
\end{aligned}
$$

Using the relations

$$
\widehat{u}_{x}^{2}-u_{x}^{2}=\left(\widehat{u}_{x}+u_{x}\right) w_{x}, \sqrt{\widehat{u}}-\sqrt{u}=\frac{w}{\sqrt{\widehat{u}+\sqrt{u}}}
$$


and Lemma 6.4, we get

$$
-I_{3} \leq \varepsilon \int_{0}^{T} \int_{0}^{a} x^{2} w_{x}^{2}+C(\varepsilon) \int_{0}^{T} \int_{0}^{a} x^{2} w^{2}+C \int_{0}^{T} \int_{0}^{a} \frac{1}{x^{4}}\left(\int_{0}^{x}|w| 3 y^{2} d y\right)^{2} .
$$

Applying Lemma 6.5 and then combining the resulting estimate for $-I_{3}$ with those for $I_{1}$ and $-I_{2}$, we deduce from (6.16) that

$$
\frac{d}{d t} \int_{0}^{T} \int_{0}^{a} x^{2} w^{2}=\int_{0}^{T} \int_{0}^{a} x^{2} w_{t} w \leq C(\varepsilon) \int_{0}^{T} \int_{0}^{a} x^{2} w^{2}
$$

provided $\varepsilon$ is small enough. It follows that $w \equiv 0$, i.e., $\widehat{u} \equiv u$.

We finally consider the asymptotic behavior of the solution, assuming that $B>0$ (if $B=0$ then $\varphi \equiv 1)$.

Let $\lambda$ and $\zeta$ be the principal eigenvalue and eigenfunction for the eigenvalue problem

$$
\begin{gathered}
\theta^{5 / 3}\left(\zeta_{x x}+\frac{2}{x} \zeta_{x}\right)+\lambda \zeta=0, \quad 0<x<a, \\
\zeta^{\prime}(0)=0, \frac{A}{2 \theta^{2 / 3}} \zeta^{\prime}(a)+\frac{B}{1+\theta} \zeta(a)=0,
\end{gathered}
$$

with $\zeta(x)>0$ if $0<x<a$ and $\zeta(a)=1$; recall that $\lambda>0$. By the maximum principle $\left(\zeta_{x x}+\frac{2}{x} \zeta_{x}<0\right)$, the minimum of $\zeta$ is positive and it is attained at $x=a$, so that

$$
\zeta(x) \geq 1
$$

THEOREM 6.6. The solution $\varphi$ of problem $\left(P 1^{\prime}\right)$ satisfies

$$
0 \leq \varphi-\theta \leq \frac{\zeta(x)}{2 \theta} e^{-\lambda \theta^{4 / 3}} t
$$

and the free boundary $x=s(t)$ satisfies (5.8).

Proof. Set $w=\varphi^{2}-\theta^{2}$ ( $w$ is $\frac{1}{2}$ times the function $w$ defined in (2.11)). We wish to compare $w$ with

$$
w_{0}=\zeta(x) e^{-\lambda \theta^{4 / 3} t} .
$$

For this purpose we introduce the operator

$$
L v=v_{t}-\frac{r^{4}}{x^{4}}\left(w+\theta^{2}\right)^{3 / 2}\left(v_{x x}+\frac{2}{x} v_{x}\right) .
$$


As seen from (3.14), (3.15),

$$
L w=\frac{4 r}{x^{2}}\left(w+\theta^{2}\right)^{3 / 2}\left(\frac{1}{\sqrt{w+\theta^{2}}}-\frac{r^{3}}{x^{3}}\right) w_{x} .
$$

From (1.4) and the inequality $\varphi_{x} \leq 0$ we get

$$
r^{3} \leq \int_{0}^{x} \frac{3 y^{2} d y}{\varphi(x, t)}=\frac{x^{3}}{\sqrt{w+\theta^{2}}} .
$$

Since also $w_{x}=2 \varphi \varphi_{x} \leq 0$, it follows that $L w \leq 0$

On the other hand

$$
\begin{gathered}
L w_{0}=\lambda w_{0}\left[-\theta^{4 / 3}+\frac{r^{4}}{x^{4}}\left(w+\theta^{2}\right)^{3 / 2} \frac{1}{\theta^{5 / 3}}\right] \\
\geq \lambda w_{0}\left[-\theta^{4 / 3}+\theta^{3} \theta^{-5 / 3}\right]=0 .
\end{gathered}
$$

On $\{x=a\},(6.10)$ implies

$$
\frac{A}{2 a^{2}} r^{2} w_{x}+B \sqrt{w+\theta^{2}}=B \theta .
$$

Since $r^{3} \leq a^{3} / \theta$ (by (6.13)) and $w>0, w_{x}<0$, we get

$$
\frac{A}{2 \theta^{2 / 3}} w_{x}+\frac{B}{1+\theta} w<0 \quad \text { at } \quad x=a .
$$

Also

$$
\frac{A}{2 \theta^{2 / 3}} w_{0, x}+\frac{B}{1+\theta} w_{0}=0 \quad \text { at } \quad x=a .
$$

Next, on the boundary $\{x=0\}, w_{x}=w_{0, x}=0$.

Finally, at $t=0, \quad w=1-\theta^{2}<1 \leq \zeta(x)=w_{0}$. Applying the maximum principle to $w_{0}-w$ we get $w_{0} \geq w$, which implies the right-hand inequality in (6.20).

Finally, (5.8) follows from (1.4) and (6.20).

Remark 6.2. One can establish Corollary 5.2 by the method of proof of Theorem 6.6.

Remark 6.3. The results of this section can be extended with minor modifications to the case where $A$ and $B$ are functions of $(r, t)$. 
§7. The case $\boldsymbol{\theta}=\mathbf{0}$. For problem (P2) the case $\theta=0$ is simply the Cauchy problem for the porous medium equation $2 \psi_{t}=\Delta \psi^{2}$. This problem has been studied extensively in the literature (see [6] and the references therein).

Consider next problems ( $\left.\mathrm{P}^{\prime}\right)$ and (P2') for small $\theta>0$ and denote the solutions by $\varphi_{\theta}$ and $\left(\psi_{\theta}, s_{\theta}\right)$. By $(6.2),(2.9)$,

$$
A \dot{s}_{\theta}=B\left(\psi_{\theta}-\theta\right) \leq B(1-\theta) .
$$

It follows that

$$
0 \leq \dot{s}_{\theta}(t) \leq C, \quad a \leq s_{\theta}(t) \leq a+C t \quad \text { for all } t>0
$$

where $C=B(1-\theta) / A$.

Lemma 7.1. For any $T>0$ there exists an $\varepsilon>0$ such that

$$
\psi_{\theta}\left(s_{\theta}(t), t\right) \geq \varepsilon^{2} \quad \text { if } \quad 0<t<T, 0<\theta<1 .
$$

Proof. Let $x_{0}=s_{\theta}\left(t_{0}\right), \quad 0<t_{0}<T$, and introduce the function

$$
W=\varepsilon\left(x_{0}-r\right)^{m}+\varepsilon^{2} \quad \text { for } \quad \frac{a}{2}<r<s_{\theta}(t), \quad 0<t<t_{0} .
$$

where $m$ is a positive integer. Then

$$
W_{t}-\frac{1}{2} \Delta W^{2}=-\frac{\varepsilon^{2}}{2} \Delta\left(x_{0}-r\right)^{2 m}-\frac{2 \varepsilon^{3}}{2} \Delta\left(x_{0}-r\right)^{m} .
$$

Clearly

$$
\Delta\left(x_{0}-r\right)^{2 m}=2 m\left(x_{0}-r\right)^{2 m-2}\left[(2 m-1)-\frac{2}{r}\left(x_{0}-r\right)\right]>0
$$

if $m$ is large enough, independently of $\varphi$ (by (7.1)), and the same holds for $\Delta\left(x_{0}-x\right)^{m}$. Hence $W$ is a subsolution: $W_{t}-\frac{1}{2} \Delta W^{2} \leq 0$.

On $r=s_{\theta}(t), 0<t<t_{0}$,

$$
A W_{r}+B W=\left(x_{0}-r\right)^{m-1} \varepsilon\left[-A m+B\left(x_{0}-r\right)\right]+B \varepsilon^{2}<0
$$

if $m$ is large (depending on $T$ ) and $\varepsilon$ is small, depending on $m$ and $T$ (both $m$ and $\varepsilon$ are independent of $\theta$ ). on $m$.

On $t=0$ we have $W<1=\psi_{\theta}(r, 0)$ if $\frac{a}{2}<r<a$ provided $\varepsilon$ is small enough depending 
Finally, in order to compare $\psi_{\theta}$ with $W$ on $r=\frac{a}{2}$ we recall [1] that there exist solutions of the porous medium equation of the form $f(r) g(t)$ for $0<r<a, t>0$ with $f(r)>$ $0, g(t)>0$ if $0 \leq r<a, t \geq 0$ such that $f(a)=0$. By comparison, $\psi_{\theta}(r, t) \geq f(r) g(t)$. Consequently

$$
\psi_{\theta}\left(\frac{a}{2}, t\right)>W\left(\frac{a}{2}\right)
$$

if $\varepsilon$ is sufficiently small (depending on $m$ and $T$ ).

We now apply comparison to deduce that $\psi_{\theta}>W$ for all $\frac{a}{2} \leq r \leq s_{\theta}(t), \quad 0 \leq t \leq t_{0}$ and, in particular, (7.2) follows at $t=t_{0}$.

From Lemma 7.1 we see that equations (2.5) and (1.9) are uniformly parabolic for $0<\theta<1,0 \leq t \leq T$, for any $T>0$. Hence the estimates established for $\varphi_{\theta}, s_{\theta}, \psi_{\theta}$ hold uniformly with respect to $\theta$. We can therefore take sequences of solutions which converge, as $\theta \rightarrow 0$, to solutions $\varphi$ and $(\psi, s)$ of $\left(\mathrm{P}^{\prime}\right)$ and $\left(\mathrm{P} 2^{\prime}\right)$ with $\theta=0$. The proof of Theorem 6.3 extends to the present case. We summarize:

ThEOREM 7.1. For $\theta=0$, there exists a solution $\varphi$ to problem $\left(\mathrm{P}^{\prime}\right)$ and a solution $(\psi, s)$ to problem $\left(\mathrm{P}^{\prime}\right)$. The solutions have the same regularity as the solutions in the case $\theta>0$, and $\varphi, \psi$ are uniformly positive for $0 \leq r \leq s(t), 0 \leq t \leq T$, for any $T>0$. Finally, the classical solutions to problems $\left(P 1^{\prime}\right)$ and $\left(P 2^{\prime}\right)$ are unique

Remark 7.1. It is clear that $s(t) \rightarrow \infty$ if $t \rightarrow \infty$. Furthermore, from the conservation law

$$
\int_{0}^{s(t)} r^{2} \psi(r, t) d r=\frac{a^{3}}{3}
$$

(which follows by integrating (2.5)) and the relations $\psi_{r}<0, \psi(s(t), t)=\frac{A}{B} \dot{s}$, it follows that

$$
s(t) \leq\left(\frac{B a^{3}}{A} t+a^{4}\right)^{1 / 4} .
$$

One can formally deduce a more precise estimate

$$
s(t) \sim \gamma t^{1 / 5} \text { as } t \rightarrow \infty
$$

where $\gamma$ is a positive constant which can be expressed in terms of $a$. Indeed, the functions $f=f_{\lambda}, \sigma=\sigma_{\lambda}$ with

$$
f_{\lambda}(r, t)=\lambda^{3} \psi\left(\lambda r, \lambda^{5} t\right), \sigma_{\lambda}(t)=\frac{\sigma\left(\lambda^{5} t\right)}{\lambda} \quad(\lambda \gg 1)
$$


solve the system

$$
\begin{aligned}
& f_{t}=\frac{1}{2} \Delta f^{2} \quad \text { if } \quad 0<r<\sigma(t), t>0 \\
& f_{r}(0, t)=0, \quad t>0 \\
& f(\sigma(t), t)=\frac{A}{B} \frac{\dot{\sigma}(t)}{\lambda}, f_{r}(\sigma(t), t)=-\dot{\sigma}(t), t>0 \\
& f(r, 0)=\lambda^{3} \quad \text { if } \quad 0<r<\frac{a}{\lambda},
\end{aligned}
$$

and

$$
\int_{0}^{\sigma(t)} r^{2} f(r, t) d r=\frac{a^{3}}{3} .
$$

If $\lambda \rightarrow \infty$ then the initial data converge to $\frac{a^{3}}{3}$ times the Dirac function whereas $f(\sigma(t), t) \rightarrow$ 0 . The solution of the limit problem is ( see [6; p. 603])

$$
\frac{b}{t^{3 / 5}}\left(1-\frac{r^{2}}{\gamma^{2} t^{2 / 5}}\right)^{+}
$$

where $b$ and $\gamma$ are determined by

$$
b=10 \gamma^{2}, 2 \gamma^{3} b=5 a^{3}
$$

Hence

$$
\sigma_{\lambda}(t)=\frac{\sigma\left(\lambda^{5} t\right)}{\lambda} \rightarrow \gamma t^{1 / 5} \quad \text { as } \quad \lambda \rightarrow \infty
$$

and (7.3) follows.

Remark 7.2. The results of this paper can be extended without difficulty to the case where the diffusion coefficient $D\left(\phi_{R}\right)$ in (1.7) is a function of $\phi_{R}$; for example, $D\left(\phi_{R}\right)=$ $\phi_{R}^{m}, m>0$.

Acknowledgement. We would like to thank Giuseppe Rossi from Ford Motor Company for suggesting the problem studied in this paper. 


\section{REFERENCES}

[1] D.G. Aronson and L.A. Peletier, Large time behavior of solutions of the porous medium equation in bounded domains, J. Diff. Eqs., 39 (1981), 378-412.

[2] R.J. Atkins and R.E. Crane, Continuum theories of mixtures: Basic theory and historical development, Quar. J. Appl. Math., 239, Part 2 (1976), 209-244.

[3] R.M. Bowen, Theory of Mixtures, in "Continuum Physics," vol. III, edited by C. Eringen, Academic Press, New York (1976), pp. 1-127.

[4] J. Crank and G.S. Park, Diffusion in Polymers, Academic Press, New York (1968).

[5] P.J. Flory, Principles of Polymer Chemistry, Cornell Univ. Press, Itacha, N.Y. (1953).

[6] A. Friedman, Variational Principles and Free-Boundary Problems, Wiley-Interscience, New York (1982).

[7] A. Friedman, Mathematics in Industrial Problems, Part 5, IMA Series, Springer-Verlag, New York (1992), to appear.

[8] A. Friedman and D. Kinderlehrer, A one-phase Stefan problem, Indiana Univ. Math. J., 24 (1975), 1005-1035.

[9] N.V. Krylov, Nonlinear Elliptic and Parabolic Equations of Second Order, Reidel, Dordrecht, The Netherlands (1987).

[10] O.A. Ladyzhenskaja, V.A. Solonnikov and N.N. Uraltseva, Linear and Quasilinear Equations of Parabolic Type, Amer. Math. Soc., Providence, R.I. (1968).

[11] G. Rossi AND K.A. MaZich, Kinetic of swelling for cross-linked elastomer or gel in the presence of a good solvent, Physical Review A, 44 (1991).

[12] D.G. SchaEffer, A new proof of the infinite differentiability of the free boundary in the Stefan problems, J. Diff. Eqs., 20 (1976), 266-269.

[13] L.R.G. Treloar, The Physics of Rubber Elasticity, Oxford University Press, Oxford (1975). 
E.G. Kalnins, Willard Miller, Jr. and Sanchita Mukherjee, Models of $q$-algebra representations: the group of plane motions

T.R. Hoffend Jr. and R.K. Kaul, Relativistic theory of superpotentials for a nonhomogeneous, spatially isotropic medium

Reinhold von Schwerin, Two metal deposition on a microdisk electrode

Vladimir I. Oliker and Nina N. Uraltseva, Evolution of nonparametric surfaces with speed depending on curvature, III. Some remarks on mean curvature and anisotropic flows

Wayne Barrett, Charles R. Johnson, Raphael Loewy and Tamir Shalom, Rank incrementation via diagonal perturbations

Mingxiang Chen, Xu-Yan Chen and Jack K. Hale, Structural stability for time-periodic one-dimensional parabolic equations

Hong-Ming Yin, Global solutions of Maxwell's equations in an electromagnetic field with the temperaturedependent electrical conductivity

Robert Grone, Russell Merris and William Watkins, Laplacian unimodular equivalence of graphs

Miroslav Fiedler, Structure-ranks of matrices

Miroslav Fiedler, An estimate for the nonstochastic eigenvalues of doubly stochastic matrices

Miroslav Fiedler, Remarks on eigenvalues of Hankel matrices

Charles R. Johnson, D.D. Olesky, Michael Tsatsomeros and P. van den Driessche, Spectra with positive elementary symmetric functions

Pierre-Alain Gremaud, Thermal contraction as a free boundary problem

K.L. Cooke, Janos Turi and Gregg Turner, Stabilization of hybrid systems in the presence of feedback delays

Robert P. Gilbert and Yongzhi Xu, A numerical transmutation approach for underwater sound propagation

LeRoy B. Beasley, Richard A. Brualdi and Bryan L. Shader, Combinatorial orthogonality

Richard A. Brualdi and Bryan L. Shader, Strong hall matrices

Håkan Wennerström and David M. Anderson, Difference versus Gaussian curvature energies; monolayer versus bilayer curvature energies applications to vesicle stability

Shmuel Friedland, Eigenvalues of almost skew symmetric matrices and tournament matrices

Avner Friedman, Bei Hu and J.L. Velazquez, A Free Boundary Problem Modeling Loop Dislocations in Crystals

Ezio Venturino, The Influence of Diseases on Lotka-Volterra Systems

Steve Kirkland and Bryan L. Shader, On Multipartite Tournament Matrices with Constant Team Size

Richard A. Brualdi and Jennifer J.Q. Massey, More on Structure-Ranks of Matrices

Douglas B. Meade, Qualitative Analysis of an Epidemic Model with Directed Dispersion

Kazuo Murota, Mixed Matrices Irreducibility and Decomposition

Richard A. Brualdi and Jennifer J.Q. Massey, Some Applications of Elementary Linear Algebra in Combinations

Carl D. Meyer, Sensitivity of Markov Chains

Hong-Ming Yin, Weak and Classical Solutions of Some Nonlinear Volterra Integrodifferential Equations

B. Leinkuhler and A. Ruehli, Exploiting Symmetry and Regularity in Waveform Relaxation Convergence Estimation

Xinfu Chen and Charles M. Elliott, Asymptotics for a Parabolic Double Obstacle Problem

Yongzhi Xu and Yi Yan, An Approximate Boundary Integral Method for Acoustic Scattering in Shallow Oceans

Yongzhi Xu and Yi Yan, Source Localization Processing in Perturbed Waveguides

Kenneth L. Cooke and Janos Turi, Stability, Instability in Delay Equations Modeling Human Respiration

F. Bethuel, H. Brezis, B.D. Coleman and F. Hélein, Bifurcation Analysis of Minimizing Harmonic Maps Describing the Equilibrium of Nematic Phases Between Cylinders

Frank W. Elliott, Jr., Signed Random Measures: Stochastic Order and Kolmogorov Consistency Conditions

D.A. Gregory, S.J. Kirkland and B.L. Shader, Pick's Inequality and Tournaments

J.W. Demmel, N.J. Higham and R.S. Schreiber, Block $L U$ Factorization

Victor A. Galaktionov and Juan L. Vazquez, Regional Blow-Up in a Semilinear Heat Equation with Convergence to a Hamilton-Jacobi Equation

Bryan L. Shader, Convertible, Nearly Decomposable and Nearly Reducible Matrices

Dianne P. O'Leary, Iterative Methods for Finding the Stationary Vector for Markov Chains

Nicholas J. Higham, Perturbation theory and backward error for $A X-X B=C$

Z. Strakos and A. Greenbaum, Open questions in the convergence analysis of the lanczos process for the real symmetric eigenvalue problem

935 Zhaojun Bai, Error analysis of the lanczos algorithm for the nonsymmetric eigenvalue problem

936 Pierre-Alain Gremaud, On an elliptic-parabolic problem related to phase transitions in shape memory alloys

937 Bojan Mohar and Neil Robertson, Disjoint essential circuits in toroidal maps 
Bojan Mohar and Svatopluk Poljak Eigenvalues in combinatorial optimization

Richard A. Brualdi, Keith L. Chavey and Bryan L. Shader, Conditional sign-solvability

Roger Fosdick and Ying Zhang, The torsion problem for a nonconvex stored energy function

René Ferland and Gaston Giroux, An unbounded mean-field intensity model:

Propagation of the convergence of the empirical laws and compactness of the fluctuations

Wei-Ming Ni and Izumi Takagi, Spike-layers in semilinear elliptic singular Perturbation Problems

Henk A. Van der Vorst and Gerard G.L. Sleijpen, The effect of incomplete decomposition preconditioning on the convergence of conjugate gradients

S.P. Hastings and L.A. Peletier, On the decay of turbulent bursts

Apostolos Hadjidimos and Robert J. Plemmons, Analysis of $p$-cyclic iterations for Markov chains

ÅBjörck, H. Park and L. Eldén, Accurate downdating of least squares solutions

E.G. Kalnins, Willard Miller, Jr. and G.C. Williams, Recent advances in the use of separation of variables methods in general relativity

G.W. Stewart, On the perturbation of LU, Cholesky and QR factorizations

G.W. Stewart, Gaussian elimination, perturbation theory and Markov chains

G.W. Stewart, On a new way of solving the linear equations that arise in the method of least squares

G.W. Stewart, On the early history of the singular value decomposition

G.W. STewart, On the perturbation of Markov chains with nearly transient states

Umberto Mosco, Composite media and asymptotic dirichlet forms

Walter F. Mascarenhas, The structure of the eigenvectors of sparse matrices

Walter F. Mascarenhas, A note on Jacobi being more accurate than QR

Raymond H. Chan, James G. Nagy and Robert J. Plemmons, FFT-based preconditioners for Toeplitz-Block least squares problems

Zhaojun Bai, The CSD, GSVD, their applications and computations

D.A. Gregory, S.J. Kirkland and N.J. Pullman, A bound on the exponent of a primitive matrix using Boolean rank

Richard A. Brualdi, Shmuel Friedland and Alex Pothen, Sparse bases, elementary vectors and nonzero minors of compound matrices

J.W. Demmel, Open problems in numerical linear algebra

James W. Demmel and William Gragg, On computing accurate singular values and eigenvalues of acyclic matrices

James W. Demmel, The inherent inaccuracy of implicit tridiagonal QR

J.J.L. Velázquez, Estimates on the $(N-1)$-dimensional Hausdorff measure of the blow-up set for a semilinear heat equation

David C. Dobson, Optimal design of periodic antireflective structures for the Helmholtz equation

C.J. van Duijn and Joseph D. Fehribach, Analysis of planar model for the molten carbonate fuel cell

Yongzhi Xu, T. Craig Poling and Trent Brundage, Source localization in a waveguide with unknown large inclusions

J.J.L. Velázquez, Higher dimensional blow up for semilinear parabolic equations

E.G. Kalnins and Willard Miller, Jr., Separable coordinates, integrability and the Niven equations

John M. Chadam and Hong-Ming Yin, A diffusion equation with localized chemical reactions

A. Greenbaum and L. Gurvits, Max-min properties of matrix factor norms

Bei Hu, A free boundary problem arising in smoulder combustion

C.M. Elliott and A.M. Stuart, The global dynamics of discrete semilinear parabolic equations

Avner Friedman and Jianhua Zhang, Swelling of a rubber ball in the presence of good solvent

Avner Friedman and Juan J.L. Velázquez, A time-dependence free boundary problem modeling the visual image in electrophotography

Richard A. Brualdi, Hyung Chan Jung and William T. Trotter, Jr., On the poset of all posets on $n$ elements

Ricardo D. Fierro and James R. Bunch, Multicollinearity and total least squares

Adam W. Bojanczyk, James G. Nagy and Robert J. Plemmons, Row householder transformations for rank- $k$ Cholesky inverse modifications

Chaocheng Huang, An age-dependent population model with nonlinear diffusion in $R^{n}$

Emad Fatemi and Faroukh Odeh, Upwind finite difference solution of Boltzmann equation applied to electron transport in semiconductor devices

Esmond G. Ng and Barry W. Peyton, A tight and explicit representation of $Q$ in sparse $Q R$ factorization

Robert J. Plemmons, A proposal for $F F T$-based fast recursive least-squares

Anne Greenbaum and Zdenek Strakos, Matrices that generate the same Krylov residual spaces

Alan Edelman and G.W. Stewart, Scaling for orthogonality

G.W. Stewart, Note on a generalized sylvester equation

G.W. Stewart, Updating URV decompositions in parallel 